\title{
Traffic and QoS-Aware Base Station Sleeping Scheme in Heterogeneous Cellular Networks
}

\author{
Lin Xiao ${ }^{1}$, Fahui $\mathrm{Wu}^{1}$, Dingcheng Yang ${ }^{1}$, Yutao $\mathrm{Zhu}^{2}$, Jietong $\mathrm{Liu}^{3}$ \\ ${ }^{1}$ Information Engineering School, Nanchang University, Nanchang, China \\ ${ }^{2}$ Beijing University of Posts and Telecommunications, Beijing, China \\ ${ }^{3}$ Queen Mary University of London, London, United Kingdom \\ xiaolin910@gmail.com; 676862214@qq.com; 53239027@qq.com; \\ yutao_bpu@126.com; jietong_qmu@126.com
}

\begin{abstract}
With the rapid development of cellular networks, wireless communication industry has brought huge energy consumption and becomes the major consumer of environmental degradation inevitably. Thus, it is undoubtedly that realizing the sustainable development of green wireless communication has become the primary goal of global information and communications technology industry. Particularly, in cellular networks, sleep mode applied to base station (BS) is an efficient way. According to the cell traffic intensity, $B S$ switching offlon strategy can reduce power consumption of BS effectively with a precondition that the quality of service $(Q o S)$ of users must be ensured. In the heterogeneous cellular networks, we proposed a BS sleeping scheme based on the traffic load varying in each cell, which is able to optimize both energy efficiency and power consumption by a multi-objective optimization problem. Then a sub-optimal solution is obtained according to a heuristic algorithm. The simulation results verified that the proposed scheme can improve the network performance.
\end{abstract}

Keywords: HetNet; sleeping scheme; traffic load; multi-objective optimization

\section{Introduction}

Due to the fact that almost $80 \%$ cellular network's total energy consumption is originating from radio access networks, the energy consumption has become a severe problem in wireless networks [1].

In the future, the cellular networks will be heterogeneous cellular networks where different types of base station (BS) in small cells are distributed throughout traditional macro cell networks [2]. Such heterogeneous cellular networks consist of different tiers of small cell networks, and the coverage of macro cell and small cell will overlap with each other, which is named as HetNets in 3GPP. In such case, the energy consumption will be more important in HetNets.

In cellular networks, the BS switching off/on (cell sleeping/waking) according to the dynamic traffic over time and space has become one of promising techniques for power saving [3-6]. The authors in [3] focused on the densely deployed cellular networks where the coverage of BSs overlaps and the traffic intensity varies over time and space. [4] optimized the energy saving assuming that any fraction of cells can be turned off. Another research on the dynamic cell sleeping in [5] used the real measured traffic data to analyze the energy consumption. The cell sleeping may lead to the network performance degradation since the remaining active BSs need to serve some users located far away from their coverage. So the authors in [6] and references therein focused on the tradeoff between the energy saving and the QoS performance. In [7], the authors focused on power matching and traffic-aware BSs sleeping. 
Obviously, energy consumption optimization by cell sleeping scheme has been researched extensively, even in the HetNets [8-11]. [8] investigated solutions for reducing the number and size of active macro cells following traffic load conditions in both homogeneous and heterogeneous networks. [9] evaluated a distributed sleep strategy for cognitive small cell BSs and analyzed the trade-off between traffic offloading from the macro cell and the energy consumption of the small cells. The authors in [10] introduced two sleep algorithms to determine the time instant to enable micro or small BSs sleep mode. [11] proposed user association jointly consider the user performance and network power saving where the cell sleeping is used for energy efficiency maximization.

The works in the literature above mainly consider one optimization target, power consumption minimization or energy efficiency maximization. However, it is essential to optimize these two targets at the same time so that cellular network could not only be green but also be efficient [12].

In this paper, a BS sleeping scheme in heterogeneous cellular networks is proposed, in which, the BS will be switched to sleep mode with low traffic load and the minimum data rate of users is guaranteed. The proposed scheme can minimize power consumption and maximize energy efficiency by a multi-objective optimization. Then a heuristic algorithm is used to solve this optimization problem and obtain a sub-optimal results. The simulation results show that the proposed scheme can reduce the power consumption and improve the energy efficiency as well.

The rest of this paper is organized as follows: Section II is the system model and the proposed scheme is presented in Section III. Section IV focuses on the simulation results, and Section V shows the conclusion of this paper.

\section{System Model}

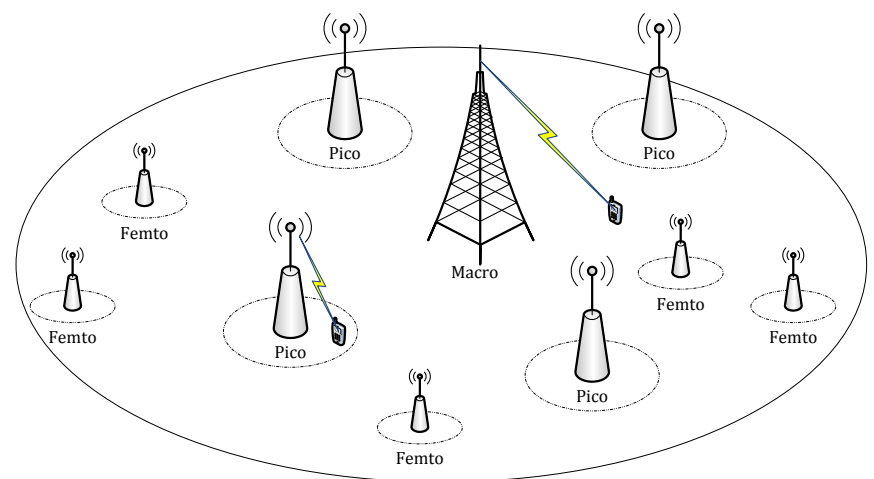

Figure 1. Example of a HetNet with a Mix of Macro, Femto and Pico BSs

In this paper, we consider downlink HetNets consisting small cells (e.g., femto and pico cells) and macro cells, where the former overlaid with the latter, as shown in Figure. 1. Assume that the system consists of cells, which contains one macro cell and small cells within the coverage of macro cell. The macro BS is deployed in the central of the macro cell. Users are included within the macro cell. Both small BSs and users are uniform distributed in the macro cell. The bandwidth of each cell is divided into subchannels and each sub-channel' bandwidth is W. These sub-channels are allocated to users according to their minimal requirement of data rate.

Since the user association is in a large time scale, the channel gain contains path loss and shadow fading, i.e., large scale fading channel. The channel gain between user $k$ and $\mathrm{BS} i$ is $g_{k, i}$.

In the macro BS $i$, the signal to interference plus noise ratio (SINR) of user $k$ is: 


$$
\gamma_{k, i}=\frac{p l_{k, i} P_{i}}{\sum_{j \neq i} p l_{k, j} P_{j}+\sigma^{2}}
$$

where $p l_{k, i}$ denotes the channel fading (including path loss and shadowing) between BS $i$ and user $k . P_{i}$ means the transmit power of the macro BS or the small BS. $\sigma^{2}$ is the noise power.

Similarly, in the small BS $i$, the SINR of user $k$ is:

$$
\gamma_{k, i}=\frac{p l_{k, i} P_{i}}{\sum_{j \neq i} p l_{k, j} P_{j}+\sigma^{2}}
$$

The data rate of signal sub-channel can be described as:

$$
C_{k, i}=W \log _{2}\left(1+\gamma_{k, i}\right)
$$

In order to ensure user's QoS which means satisfying the minimal data rate of each user, the number of required sub-channels $Q_{k, i}$ can be formed as:

$$
Q_{k, i}=\left\lceil\frac{\breve{r}_{k}}{C_{k, i}}\right\rceil
$$

On the one hand, the number of sub-channels must be integer. On the other hand, it is necessary to guarantee the user performance. Thus, $Q_{k, i}$ is taken as rounding up to an integer.

The traffic load for user $k$ associated with BS $i$ could be defined as the ratio of occupied channels and total channels:

$$
L_{k, i}=\frac{Q_{k, i}}{N}
$$

The traffic load of BS $i$ is $L_{i}$. It is the total sum of each traffic load of users who access this BS. So the corresponding mathematical expression is:

$$
L_{i}=\sum_{k=1}^{K} \lambda_{k, i} L_{k, i}
$$

in which, $\lambda_{k, i}$ is the indicator variable to represent whether user $k$ associated with BS $i$ or not, that is:

$$
\lambda_{k, i}=\left\{\begin{array}{l}
1, \text { user } k \text { associated with BS } i \\
0, \text { otherwise }
\end{array}\right.
$$

The throughput of BS $i, R_{i}$ is defined as the sum of transmission rate of UEs associated with this BS:

$$
R_{i}=\sum_{k=1}^{K} \lambda_{k, i} r_{k, i}=\sum_{k=1}^{K} \lambda_{k, i} Q_{k, i} C_{k, i}
$$

and the throughput of the whole network is:

$$
R_{\text {total }}=\sum_{i=1}^{B} R_{i}
$$

When BS is in sleep mode, only static circuit power will consume in BS. For BS $i$, power consumption is:

$$
P_{i n}(i)=P_{s}(i), L_{i}=0
$$

where $P_{s}(i)$ is static circuit power consumption of BS $i$.

When BS is in active mode, the power consumption is much more complicated. For BS $i$, power consumption is:

$$
P_{\text {in }}(i)=P_{a}(i)+\beta_{i} R_{i}+\alpha_{i} L_{i} P_{\text {max }}(i), 0<L_{i} \leq 1
$$


The power consumption can be separated into three parts. First, BS $i$ consumes static circuit power $P_{a}(i)$ to be active. Second, dynamic power $\beta_{i} R_{i}, \beta_{i}$ is circuit power of one unit data rate of BS $i$. By multiplying the transmission rate of the BS, the total dynamic power consumption can be gotten. Third, power $\alpha_{i} L_{i} P_{\max }(i)$ from PA, $\alpha_{i}$ is the PA efficiency of BS $i$, the maximal transmission rate is used when the traffic load is full, but in most cases, it is not rigorous to use $P_{\max }(i)$ directly. Hence, $L_{i}$ is introduced to make it precise. Particularly, $P_{s}(i), P_{a}(i), \beta_{i}$ and $P_{\max }(i)$ are different for the reason that macro BS and micro BS are different.

It can be seen that the total power consumption is:

$$
P_{\text {total }}=\sum_{i=1}^{B} P_{i n}(i)
$$

According to the definition of energy efficiency in [12], the energy efficiency is equal to the ratio of system throughput and total power consumption:

$$
E E=\frac{R_{\text {total }}}{P_{\text {total }}}
$$

The multi-objective optimization problem (MOP) is modeled as:

$$
\left\{\begin{array} { l } 
{ \operatorname { m i n } _ { \lambda _ { k , i } , r _ { k , i } } P _ { \text { total } } } \\
{ \operatorname { m a x } _ { \lambda _ { k , i } , r _ { k , i } } E E }
\end{array} \quad \text { s.t } \left\{\begin{array}{l}
r_{k, i} \geq \breve{r}_{k}, \forall k \\
\lambda_{k, i} \in\{0,1\} \\
\sum_{i=1}^{B} \lambda_{k, i}=1 \\
0<L_{i} \leq 1
\end{array}\right.\right.
$$

Actually, for the second object, it can be regarded as enlarging the whole network transmission rate and reducing the power. Therefore, the optimization targets equivalent to:

$$
\left\{\begin{array} { l } 
{ \operatorname { m i n } _ { \lambda _ { k , i } , r _ { k , i } } P _ { \text { total } } } \\
{ \operatorname { m a x } _ { \lambda _ { k , i } , r _ { k , i } } R _ { \text { total } } }
\end{array} \quad \text { s.t } \left\{\begin{array}{l}
r_{k, i} \geq \breve{r}_{k}, \forall k \\
\lambda_{k, i} \in\{0,1\} \\
\sum_{i=1}^{B} \lambda_{k, i}=1 \\
0<L_{i} \leq 1
\end{array}\right.\right.
$$

From [13], the MOP could be solved as single objective optimization problem when $R_{\text {total }}$ is given. Apply the lemma 1 in [13] which states that for a given $R_{\text {total }}^{*}$, if $P_{\text {total }}^{*}$ is an optimal solution for optimizing power consumption, then $\left(R_{\text {total }}^{*}, P_{\text {total }}^{*}\right.$ ) is a Pareto solution to MOP, and corresponding solution is a suboptimal solution to MOP. The detail proof is shown in [13]. For convenience and satisfying the requirement of QoS simultaneously, $r_{k, i}$ is set equivalent to $\breve{r}_{k}$. By this way, a fixed $R_{\text {total }}$ is given, and the design target is only to minimize $P_{\text {total }}$ :

$$
\begin{aligned}
& \min _{\lambda_{k, i}} P_{\text {total }} \\
& \text { s.t }\left\{\begin{array}{l}
r_{k, i}=\breve{r}_{k}, \forall k \\
\lambda_{k, i} \in\{0,1\} \\
\sum_{i=1}^{B} \lambda_{k, i}=1 \\
0<L_{i} \leq 1
\end{array}\right.
\end{aligned}
$$




\section{Sub-optimal Solution}

From previous equation (11), as a result of active static circuit power $P_{a}(i)$, dynamic circuit power efficiency $\beta_{i}$, power amplifier efficiency $\alpha_{i}$ and maximal transmit power $P_{\max }(i)$ are fixed for a specified BS. Then we know that power consumption is only relating to traffic load since transmission rate is fixed as lowest user requirement. For convenience, minimal transmission rate requirement for all the users is assumed to be same, and then throughput of each BS and the whole network is known. Thus, once distribution of users and BSs are determined, the problem can be solved.

First, calculate SNR, a $K \times B$ matrix. Then substituting formulation, traffic load is also a $K \times B$ matrix. By summing all values of each column, the traffic load of each BS can be obtained. At this moment, BS that takes smallest traffic load will save most power consumption. In terms of this BS, pick out the user who brings least traffic load, which implies best SINR, to try to access corresponding BS. In this way, not only can users get best QoS but also BSs will consume lowest power. Once this user gets the service, next one with lowermost in the rest user set is able to repeat the process.

When a user considers accessing to a BS, it is necessary to make a judgment whether this BS will overload if this user join in. Because we have calculated potential traffic load user may bring to this BS, it is convenient and efficient to estimate whether the sum of existing traffic load and acquiring load will exceed BS's ability. This condition can be written as $\operatorname{load}(b)+\operatorname{load}(k, b) \leq 1$. If the appointed BS will overload, user will give up and choose other BS s to access. In the remaining BSs, same method can be used.

The details of the proposed scheme according to the above heuristic idea is given as follows:

Step 1: Initialize system parameters. BSs set is defined as $C=\{1,2, \cdots, B\}$ and users set is $U=\{1,2 \cdots, K\}$;

Step 2: Calculate traffic load $\operatorname{load}(k, b)$ for every user that needs to access with the given transmission rate;

Step 3: Calculate the sum of each BS's traffic load, $\operatorname{load}(b)=\operatorname{sum}(\operatorname{load}(:, b))$, $\forall k \in U, \forall b \in C$;

Step 4: Choose BS that has smallest traffic load $\hat{b}=\underset{b \in C}{\operatorname{argmin}} \operatorname{load}(b)$;

Step 5: Sort users in set $U$ by $\operatorname{load}(k, \hat{b})$ in ascending order, and let user access BS $\hat{b}$ in accord with this order until the BS is full;

Step 6: Delete BS $\hat{b}$ and users who have accessed from set $C$ and $U$ respectively;

Step 7: Repeat Step 3 until set $C$ or $U$ is empty.

The above process in the scheme considers a static user distribution in the networks. However, it may have new users access into the networks or some users leave the networks. So if there some new users arrive, it is possible to keep the accessed users unchanged and these new users are able to access to BSs. Then the remaining BSs have no traffic load and they can be put into sleep mode, which only consume small static circuit power.

If active BSs are not enough to satisfy all new users, some BSs in sleep mode ought to be aroused to provide service. In terms of the rule how to waking up BS, the details are given as follows. 
Step 1: Obtain new system parameters. New users set is $E=\{1,2 \cdots, K\}$, active BSs set is $A=\{1,2, \cdots, M\}$, the sleep BSs set is $S=\{1,2, \cdots, N\}$ and $M+N=B$;

Step 2: Calculate traffic load $\operatorname{load} 0(k, b)$ for every user that needs to access with the given transmission rate;

Step 3: Obtain the sum of each active BS's traffic load at last moment, $\operatorname{load}(b)=\operatorname{sum}(\operatorname{load}(:, b)), \forall k \in E, \forall b \in A$;

Step 4: Choose active BS that has smallest traffic load $\hat{b}=\underset{b \in A}{\operatorname{argmin}} \operatorname{load}(b)$;

Step 5: Sort users in set $E$ by $\operatorname{load} 0(k, \hat{b})$ in ascending order, and let user access BS $\hat{b}$ in accord with this order until the BS is full;

Step 6: Delete BS $\hat{b}$ and users who have accessed from set $A$ and $E$ respectively;

Step 7: Repeat Step 3 until set $E$ or $A$ is empty. If $E$ is empty, end; otherwise, go to Step 8;

Step 8: Calculate the sum of each sleep BS's traffic load, $\operatorname{load}(b)=\operatorname{sum}(\operatorname{load}(:, b))$;

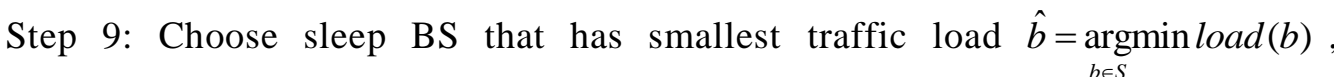
$\forall k \in E, \forall b \in S$;

Step 10: Sort users in set $E$ by $\operatorname{load} 0(k, \hat{b})$ in ascending order, and let user access BS $\hat{b}$ in accord with this order until the BS is full;

Step 11: Delete BS $\hat{b}$ and users who have accessed from set $S$ and $E$ respectively;

Step 12: Repeat Step 8 until set $E$ or $S$ is empty.

\section{Simulation Results and Analysis}

To verify the proposed sleeping scheme, the simulation results are given. We consider a HetNet with one macro cell and multiple micro cells (can be seen as a kind of small cells). The simulation parameters are presented in Table 1.

Table 1. Simulation Parameters

\begin{tabular}{|l|c|}
\hline \multicolumn{1}{|c|}{ Parameter } & Value \\
\hline The number of BSs & 10 \\
\hline Coverage of macro BS & $0.5 \mathrm{~km}^{2}$ \\
\hline The number of subchannel for each BS & 25 \\
\hline The bandwidth of single sub-channel & $200 \mathrm{kHz}$ \\
\hline Static circuit power of macro BS in sleep mode & $558 \mathrm{~W}$ \\
\hline Static circuit power of micro BS in sleep mode & $78 \mathrm{~W}$ \\
\hline Maximal transmit power of macro BS & $40 \mathrm{~W}$ \\
\hline Maximal transmit power of micro BS & $6.3 \mathrm{~W}$ \\
\hline Static circuit power of macro BS in active Mode & $712 \mathrm{~W}$ \\
\hline Static circuit power of micro BS in active mode & $106 \mathrm{~W}$ \\
\hline Power amplifier efficiency of macro BS & 14.5 \\
\hline Power amplifier efficiency of micro BS & 6.35 \\
\hline Dynamic circuit power efficiency & $2 \mathrm{~W} / \mathrm{Mbps}$ \\
\hline
\end{tabular}




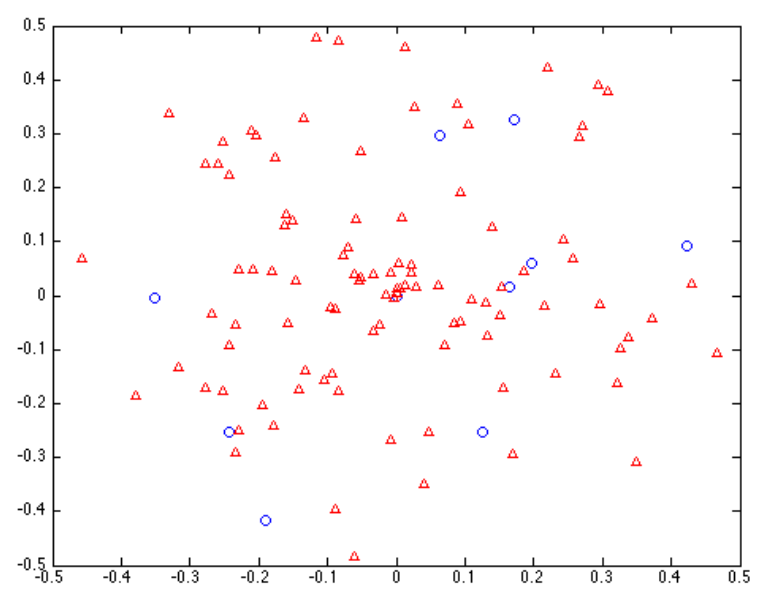

Figure 2. Micro BS and User Distribution within a Macro Cell

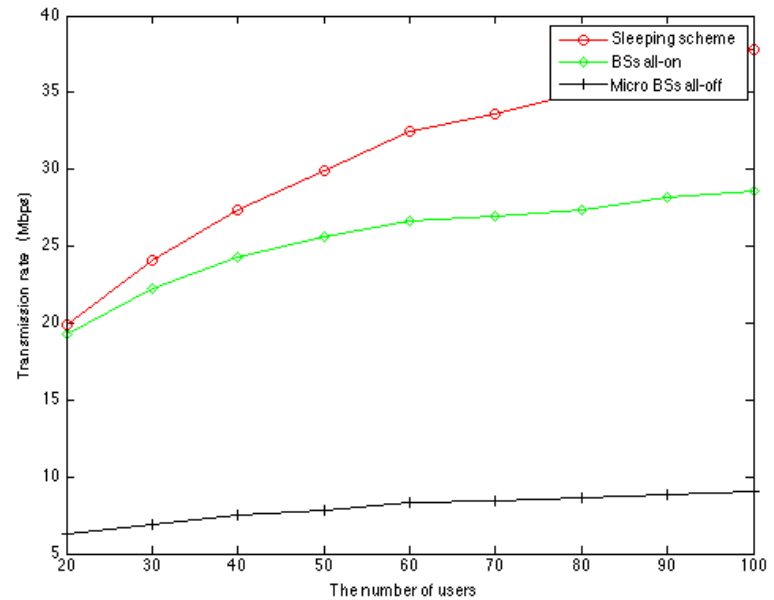

Figure 3. Data Rate of Users' Comparison varying with User Numbers

The BS and user distribution is shown in Figure 2. Within a macro cell's coverage, these blue circles stand for micro BSs and red triangles stand for users. We assume not only micro BSs but also users distribute uniformly and randomly.

Figure. 3 shows the results of data rate of users' comparison varying with user numbers. Red line represents the proposed scheme, green line represents BSs all-on strategy and black line represents Micro BSs all-off method.

The total transmission data rate of the whole network of Micro BSs all-off is the lowest. Because the capacity limitation of single macro BS. When users are too many, macro BS has no free sub-carriers for them. Therefore, macro BS has to abandon remaining users. As for BSs all-on, since the access priority is according to every user's best SNR performance. This seems to be beneficial for user but not for the whole network. In terms of sleeping scheme, the first indicator is best SNR in global range. So less resource will be accounted. At initial with little users, BSs all-on and sleeping scheme seems to be the same, but in later part, sleeping scheme increased more than former while both of them will become steady gradually. 


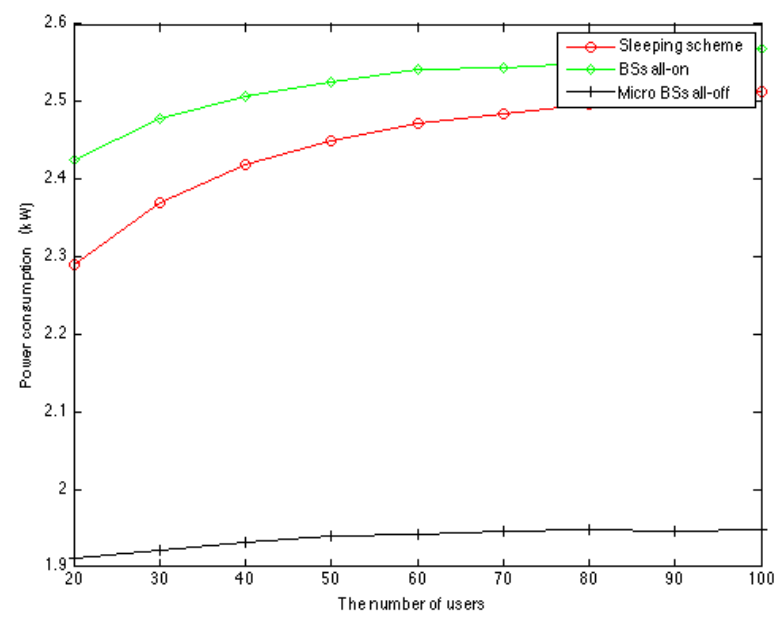

Figure 4. Power Consumption Comparison varying with User Numbers

Then the power consumption is presented in Figure 4. It is evident that power consumption of all these three schemes shows a growth trend with the rise of users. Only macro BS consumes power in micro BSs all-off, its value is minimal. More importantly, when users' requirements are out of its ability, macro BS is full then its power consumption is going to be a constant. As a fact that all BSs stay in active mode will expend more power to maintain normal operation, there is no deny that it depletes the most power. In the proposed sleeping scheme, sleep mode will save much unnecessary energy waste as well as ensuring the QoS of users.

Figure. 5 indicates the energy efficiency of the networks. Obviously, under the same transmission rate requirement, the proposed sleeping 9scheme has a better energy efficiency performance than other two. With the increasing of the number of users, turning off all micro BSs are going to ascend slowly. This is purely because there is only macro BS keeping on working and its capacity is confined, which leads to only a part of users are able to get service from it. In terms of BSs all-on, as a result of power consumption of it is the highest one, which will decrease the energy efficiency.

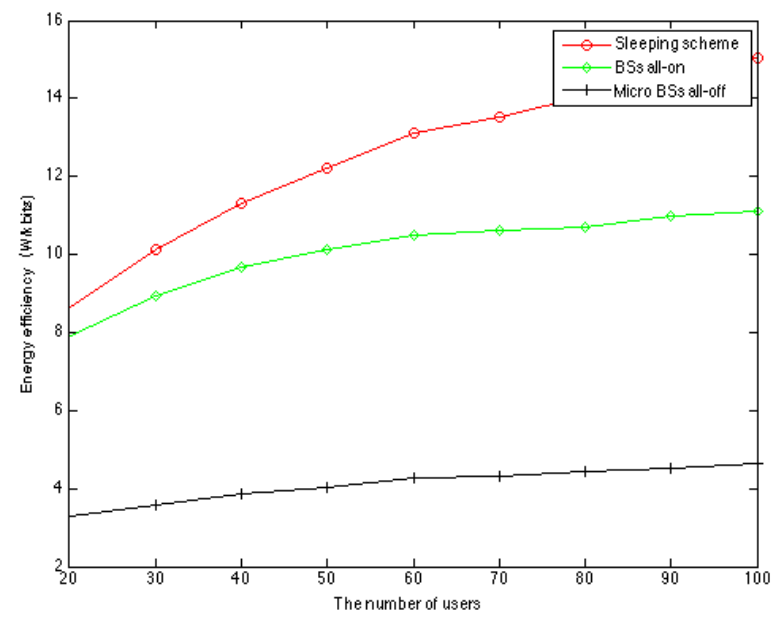

Figure 5. Energy Efficiency comparison varying with User Numbers 


\section{Conclusions}

In this paper, a BS sleeping scheme is proposed in the HetNets. The MOP is modeled as, power consumption minimization and energy efficiency maximization, which can make the networks green and efficient. Then a heuristic sleeping scheme with low complexity is designed to solve the MOP. The simulation results are obtained, which verify that the proposed BS sleeping scheme can improve the network throughput, reduce power consumption, and enhance energy efficiency as well.

\section{References}

[1] J. G. Andrews, H. Claussen, M. Dohler, S. Rangan and M. C. Reed, "Femtocells: Past, Present, and Future", Selected Areas in Communications, IEEE Journal, vol. 30, no. 3, (2012), pp. 497-508.

[2] G. Koutitas, A. Karousos and L. Tassiulas, "Deployment Strategies and Energy Efficiency of Cellular Networks", in Wireless Communications, IEEE Transactions, vol. 11, no. 7, (2012), pp. 2552-2563.

[3] J. Gong, S. Zhou, Z. Niu and P. Yang, "Traffic-aware base station sleeping in dense cellular networks", in Quality of Service (IWQoS), 2010 18th International Workshop, (2010), pp. 1-2.

[4] M. A. Marsan, L. Chiaraviglio, D. Ciullo and M. Meo, "Optimal Energy Savings in Cellular Access Networks", in IEEE International Conference on Communications Workshops, (2009), pp. 1-5.

[5] E. Oh, B. Krishnamachari, X. Liu and Z. Niu, "Toward dynamic energy-efficient operation of cellular network infrastructure", Communications Magazine, IEEE, vol. 49, no. 6, (2011), pp. 56-61.

[6] Y. Zhu, T. Kang, T. Zhang and Z. Zeng, "QoS-aware user association based on cell zooming for energy efficiency in cellular networks", in Personal, Indoor and Mobile Radio Communications (PIMRC Workshops), 2013 IEEE 24th International Symposium, (2013), pp. 6-10.

[7] Y. Wu, N. B. Shroff and Z. Niu, "Energy minimization in cooperative relay networks with sleep modes", in Modeling and Optimization in Mobile, Ad Hoc and Wireless Networks (WiOpt), 2012 10th International Symposium, (2012), pp. 200-207.

[8] C. Khirallah, J. S. Thompson and D. Vukobratovic, "Energy efficiency of heterogeneous networks in LTE-advanced", in Wireless Communications and Networking Conference Workshops (WCNCW), 2012 IEEE, (2012).

[9] M. Wildemeersch, T. Q. S. Quek, C. H. Slump and A. Rabbachin, "Cognitive Small Cell Networks: Energy Efficiency and Trade-Offs", in Communications, IEEE Transactions, vol. 61, no. 9, (2013), pp. 4016-4029.

[10] P. Dini, P. Miozzo, M. Bui and N. Baldo, "A Model to Analyze the Energy Savings of Base Station Sleep Mode in LTE HetNets", in Green Computing and Communications (GreenCom), 2013 IEEE and Internet of Things (iThings/CPSCom), IEEE International Conference on and IEEE Cyber, Physical and Social Computing, (2013), pp. 1375-1380.

[11] Y. Zhu, Z. Zeng, T. Zhang, L. An and L. Xiao, "An energy efficient user association scheme based on cell sleeping in LTE heterogeneous networks", in Wireless Personal Multimedia Communications (WPMC), 2014 International Symposium, (2014), pp. 75-79.

[12] J. Cao, T. Zhang and Z. Zeng, "Multi-relay selection schemes based on evolutionary algorithm in cooperative relay networks", International Journal of Communication Systems, vol. 27, no. 4, (2014), pp. 571-591.

[13] C. He, B. Sheng, P. Zhu and X. You, "Energy Efficiency and Spectral Efficiency Tradeoff in Downlink Distributed Antenna Systems", in Wireless Communications Letters, IEEE, vol. 1, no. 3, (2012), pp. 153-156. 
International Journal of Future Generation Communication and Networking Vol. 9, No.9, (2016) 